\title{
An experimental investigation of criteria for continuous variable entanglement
}

\author{
W. P. Bowen, R. Schnabel, and P. K. Lam \\ Department of Physics, Faculty of Science, Australian National University, ACT 0200, Australia \\ T. C. Ralph \\ Department of Physics, Centre for Quantum Computer Technology, \\ University of Queensland, St Lucia, QLD 4072, Australia
}

\begin{abstract}
We generate a pair of entangled beams from the interference of two amplitude squeezed beams. The entanglement is quantified in terms of EPR-paradox [1] and inseparability[2] criteria, with observed results of $\Delta^{2} X_{x \mid y}^{+} \Delta^{2} X_{x \mid y}^{-}=0.58 \pm 0.02$ and $\sqrt{\Delta^{2} X_{x \pm y}^{+} \Delta^{2} X_{x \pm y}^{-}}=0.44 \pm 0.01$, respectively. Both results clearly beat the standard quantum limit of unity. We experimentally analyze the effect of decoherence on each criterion and demonstrate qualitative differences. We also characterize the number of required and excess photons present in the entangled beams and provide contour plots of the efficacy of quantum information protocols in terms of these variables.
\end{abstract}

PACS numbers: 42.50.Dv, 42.65.Yj, 03.67.Hk

Entanglement is one of the most interesting properties of quantum mechanics, and is an important ingredient of quantum information protocols such as quantum teleportation[3], densecoding [4, 5] and quantum computation [6]. In the Schrödinger picture, a necessary and sufficient criterion for the emergence of entanglement is that the state describing the entire system is inseparable, i.e. the wavefunction of the total system cannot be factored into a product of separate contributions from each sub-system. Using the Heisenberg approach, a sufficient criterion for the presence of entanglement is that correlations between conjugate observables of two subsystems allow the statistical inference of either observable in one sub-system, upon a measurement in the other, to be smaller than the standard quantum limit, i.e. the presence of non-classical correlations. The latter approach was originally proposed in the paper of Einstein, Podolsky and Rosen [7]. These two different pictures result in two distinct methods of characterizing entanglement. One is to identify an observable signature of the mathematical criterion for wave-function entanglement, i.e. inseparability of the state. The second looks directly for the onset of non-classical correlations. For pure states these two approaches return the same result suggesting consistency of the two methods. However, when decoherence is present, causing the state to be mixed, differences can occur.

We present the generation and investigation of Gaussian continuous variable entanglement between the amplitude and phase quadratures of a pair of light beams; henceforth termed quadrature entanglement. We will use the measure proposed by Duan et al. [2] as an example that identifies wave-function inseparability and refer to this as the inseparability criterion. As an example of an entanglement criterion based directly on the observation of non-classical correlations, we will use the demonstration of the EPR-paradox as quantified by Reid and Drummond [1], and refer to this as the EPR criterion. We introduce decoherence in the form of optical loss to both entangled beams and experimentally demonstrate qualitative and quantitative differences between the two criteria. These dif-

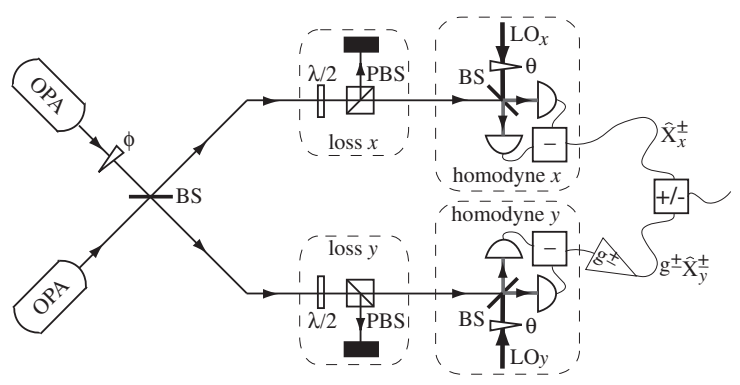

FIG. 1: Experimental schematic. BS (PBS): 50/50 (polarizing) beam splitter, $\lambda / 2$ : half-wave plate, $\phi$ and $\theta$ : phase shift.

ferences suggest a more comprehensive representation may be appropriate. We represent the entanglement on a plot of the number of photons required to generate it versus the number of excess photons. We present efficacy contours for some common quantum information protocols on this plot.

Fig. 11 shows the experimental setup that was used to generate quadrature entanglement. Two independent amplitude squeezed beams at $1064 \mathrm{~nm}$ were produced by a pair of type I optical parametric amplifiers (OPAs) constructed from hemilithic $\mathrm{MgO}: \mathrm{LiNbO}_{3}$ crystals and output couplers. The seed power to the OPAs was adjusted so that the two squeezed beams were of equal intensity. Both had $4.1 \mathrm{~dB}$ of squeezing at $6.5 \mathrm{MHZ}$ as measured in a homodyne detector with total detection efficiency of $85 \%$. Further details of this setup are reported in [8]. We produced a pair of quadrature entangled beams by interfering the squeezed beams with relative phase of $\pi / 2$ on a $50 / 50$ beam splitter, and observed a visibility of $98.7 \pm 0.3 \%$ for the process. Each of the entangled beams was interrogated in a balanced homodyne detector that could be locked to detect either its phase or amplitude quadrature. We observed $96 \pm 0.5 \%$ visibility between each entangled beam and its homodyne local oscillator. Epitaxx ETX500 photodiodes with approximately $93 \%$ quantum efficiency were used. Both the EPR and inseparability criteria could be quantified 
by analyzing correlations between these two homodynes.

The EPR criterion for a given pair of beams $x$ and $y$ can be related to their degree of quantum correlation. This is quantified by the product of conditional variances $\Delta^{2} \hat{X}_{x \mid y}$ of conjugate quadratures between the beams. Limiting ourselves to the amplitude $\hat{X}^{+}$and phase $\hat{X}^{-}$quadratures, the EPR criterion is [1]

$$
\Delta^{2} \hat{X}_{x \mid y}^{+} \Delta^{2} \hat{X}_{x \mid y}^{-}<1
$$

where $\Delta^{2} \hat{X}_{x \mid y}^{ \pm}=\Delta^{2} \hat{X}_{x}^{+}-\left|\left\langle\delta X_{x}^{ \pm} \delta X_{y}^{ \pm}\right\rangle\right|^{2} / \Delta^{2} \hat{X}_{y}^{ \pm}=$ $\min _{g^{ \pm}}\left\langle\left(\delta X_{x}^{ \pm}-g^{ \pm} \delta X_{y}^{ \pm}\right)^{2}\right\rangle$; we expand the quadrature operators into a mean unchanging term $\left\langle\hat{X}^{ \pm}\right\rangle$and a noise operator $\delta \hat{X}^{ \pm}, \hat{X}^{ \pm}=\left\langle\hat{X}^{ \pm}\right\rangle+\delta \hat{X}^{ \pm}$. Satisfaction of the EPR criterion between two beams is a sufficient but not necessary condition for their entanglement (i.e inseparability). It is easily measurable, and has been used to characterize entanglement in a number of experiments [9].

We are interested in the effect of decoherence in the form of optical loss on the EPR and inseparability criteria. For entanglement generated as in fig. 1 from a pair of pure squeezed beams both with squeezed variances $\Delta^{2} \hat{X}_{\mathrm{sqz}}$, the left-handside of eq. (1) as a function of detection efficiency $\eta$ is

$$
\Delta^{2} \hat{X}_{x \mid y}^{+} \Delta^{2} \hat{X}_{x \mid y}^{-}=4\left(1-\eta+\frac{2 \eta-1}{\eta\left(\Delta^{2} \hat{X}_{\mathrm{sqz}}+\Delta^{2} \hat{X}_{\mathrm{sqz}}^{-1}-2\right)+2}\right)^{2}
$$

Notice that when $\eta=0.5, \Delta^{2} \hat{X}_{x \mid y}^{+} \Delta^{2} \hat{X}_{x \mid y}^{-}=1$, independent of the level of squeezing; it follows that for $\eta<0.5$ the EPR criterion cannot hold.

The inseparability criterion relies on the identification of separability with positivity of the P-representation distribution of the state. For states with Gaussian noise distributions and symmetric correlations on the conjugate quadratures, it can be related to measurable correlations [2]

$$
\left\langle\left(|a| \hat{X}_{x}^{+}+\frac{\hat{X}_{y}^{+}}{a}\right)^{2}\right\rangle+\left\langle\left(|a| \hat{X}_{x}^{-}-\frac{\hat{X}_{y}^{-}}{a}\right)^{2}\right\rangle<2\left(a^{2}+\frac{1}{a^{2}}\right)
$$

where $a$ is an experimentally adjustable parameter. In our experiment the entangled beams were produced on a 50/50 beam splitter and were indistinguishable from each other; in this case $a=1$ and the criterion can be written $\Delta^{2} \hat{X}_{x \pm y}^{+}+$ $\Delta^{2} \hat{X}_{x \pm y}^{-}<2$, where $\Delta^{2} \hat{X}_{x \pm y}$ is the minimum of the variance of the sum or difference of the operator $\hat{X}$ between beams $x$ and $y$ normalized to the two beam shotnoise, $\Delta^{2} \hat{X}_{x \pm y}=$ $\min \left\langle\left(\delta \hat{X}_{x} \pm \delta \hat{X}_{y}\right)^{2}\right\rangle / 2$. The symmetric correlation restriction on eq. (3) becomes unnecessary for indistinguishable entangled beams when the criterion is written in a product form

$$
\sqrt{\Delta^{2} \hat{X}_{x \pm y}^{+} \Delta^{2} \hat{X}_{x \pm y}^{-}}<1
$$

For our experimental configuration the left-hand-side of eq. (4) as a function of efficiency can be expressed

$$
\sqrt{\Delta^{2} \hat{X}_{x \pm y}^{+} \Delta^{2} \hat{X}_{x \pm y}^{-}}=\eta \Delta^{2} \hat{X}_{\mathrm{sqz}}+(1-\eta)
$$

Notice that, in contrast to the EPR criterion, even as $\eta$ approaches zero, for any level of squeezing eq. (5) is below unity. It can be shown from eqs. (2) and (5) however, that for $\eta=1$, (i.e. for pure measured states) criteria (11) and (\#) are qualitatively equivalent.

In our experiment correlations were observed between the output currents from the homodyne detectors measuring beams $x$ and $y$ when locked simultaneously to the amplitude or phase quadrature. The inseparability criterion was determined by analyzing the unity gain sum and difference photocurrents between the two homodynes in a spectrum analyzer. The spectrum analyzer had $300 \mathrm{kHz}$ resolution bandwidth and $300 \mathrm{~Hz}$ video bandwidth and was set to zero span at $6.5 \mathrm{MHz}$. Ten consecutive traces each containing 400 measurement points were averaged for each result. All measured traces were at least $4.5 \mathrm{~dB}$ above the detection darknoise which was taken into account. The variances of the sum and difference photocurrents were normalized to the vacuum noise scaled by the combined power of the two homodyne local oscillators and the two entangled beams. The minimum of these two normalized variances is $\Delta_{x \pm y}^{2} \hat{X}$ for the quadrature being measured in the homodynes. With no additional loss in the system we observed $\Delta^{2} \hat{X}_{x \pm y}^{ \pm}=0.44 \pm 0.01$ for both the amplitude and phase quadratures. The left-hand-side of criterion (4) was then $\sqrt{\Delta^{2} \hat{X}_{x \pm y}^{+} \Delta^{2} \hat{X}_{x \pm y}^{-}}=0.44 \pm 0.01$ which is well below unity and indicates that our beams were indeed quadrature entangled.

The EPR criterion was determined in a similar manner to that described above. This time, however, rather than being fixed to unity, the gain between the two homodyne photocurrents was optimized to minimize the measured variances; and the normalization was performed with respect to vacuum fluc-

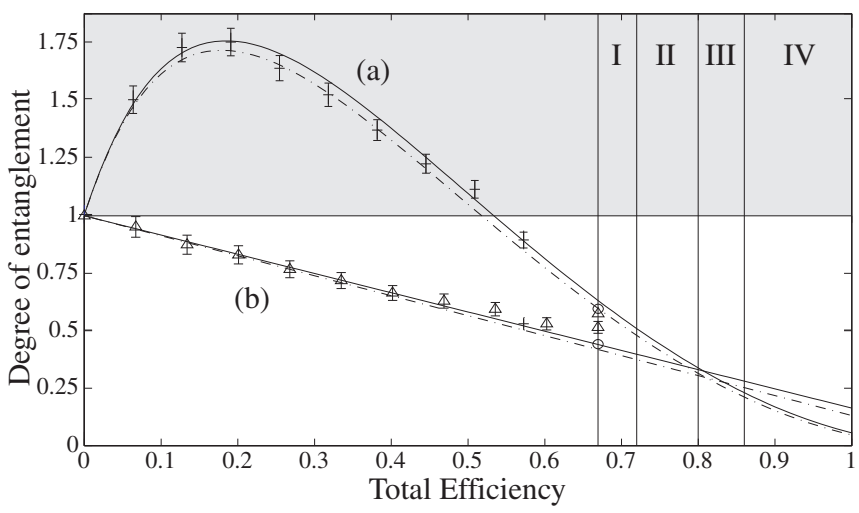

FIG. 2: Comparison of (a) EPR and (b) inseparability criteria with varied detection efficiency. The symbols,$+ \triangle$, and $\bigcirc$ label three separate experimental runs. For + a systematic error was introduced by the detection darknoise when optimizing the EPR criterion gain. The solid fit in (a) includes this, the dashed fit is the result expected if the error was eliminated, and agrees well with runs $\triangle$ and $\bigcirc$. The solid line in (b) is a theoretical fit, the dashed line is the result predicted by the fit in (a). There were four sources of unavoidable loss in our system, I: Detection loss, II: Homodyne loss, III: optical loss and IV: OPA escape loss. 
tuations scaled by only one homodyne local oscillator and entangled beam. The optimum results obtained for each quadrature were $\Delta^{2} \hat{X}_{x \mid y}^{+}=0.77 \pm 0.01$ and $\Delta^{2} \hat{X}_{x \mid y}^{-}=0.76 \pm 0.01$, and the left-hand-side of criterion (1) was in the regime for observation of the EPR-paradox, $\Delta^{2} \widehat{X}_{x \mid y}^{+} \Delta^{2} \hat{X}_{x \mid y}^{-}=0.58 \pm 0.02<1$.

As discussed earlier, the criterion for demonstration of the EPR-paradox and inseparability are qualitatively different. In particular, the criterion for demonstration of the EPR-paradox strongly depends on mixedness, whereas the inseparability criterion is independent of it. We demonstrated this experimentally by adding loss to each of the quadrature entangled beams. This changed both the degree of entanglement and also it's mixedness. Placing a polarizing beam splitter and half-wave plate in each entangled beam allowed the introduction of arbitrary loss. We took measurements of both criteria for a number of loss settings, the results are shown in fig. 2. The experiment agrees very well with our theoretical predictions. As discussed in [2], no matter what the loss, the inseparability criterion always holds. The EPR criterion however, fails for loss greater than 0.48. In fact as observed earlier, it is impossible for the EPR criterion to hold for loss greater than or equal to 0.5 . The error bars on the plots can be attributed to uncertainty in the loss introduced, small fluctuations in the local oscillator powers and, for the EPR criterion, error in the optimization of the electronic gain.

Many applications have been proposed for entanglement. In general, these applications differ in their sensitivity to the mixedness of the entanglement. For example, unity gain quantum teleportation is independent of mixedness, whereas densecoding can be very sensitive to it. For this reason, it is useful to characterize entanglement on a two dimensional diagram which includes both the degree of entanglement and its mixedness. This may be conveniently achieved with a photon number diagram.

The average number of photons per unit bandwidth per unit time in a sideband of an optical beam is given by $\bar{n}=\left\langle\delta \hat{a}^{\dagger} \delta \hat{a}\right\rangle$, where $\hat{a}$ is the field annihilation operator and can be related to the quadrature operators by $\hat{a}=\left(\hat{X}^{+}+i \hat{X}^{-}\right) / 2$. $\bar{n}$ can be written in terms of quadrature variances as $\bar{n}=\left(\Delta^{2} \hat{X}^{+}+\right.$ $\left.\Delta^{2} \hat{X}^{-}-2\right) / 4$. In general some minimum number of photons $\bar{n}_{\text {min }}$ is required to maintain a given degree of entanglement between a pair of optical beams. For indistinguishable entanglement with Gaussian noise statistics $\bar{n}_{\text {min }}$ can be expressed as a function of the left-hand-side of the inseparability criterion in eq. (4)

$$
\bar{n}_{\min }=\frac{\left(\Delta^{2} \hat{X}_{x \pm y}^{+} \Delta^{2} \hat{X}_{x \pm y}^{-}\right)^{\frac{1}{2}}+\left(\Delta^{2} \hat{X}_{x \pm y}^{+} \Delta^{2} \hat{X}_{x \pm y}^{-}\right)^{-\frac{1}{2}}}{2}-1
$$

and is a measure of the degree of entanglement. Any photons in excess of $\bar{n}_{\min }$ are not necessary for the entanglement and contribute to the mixedness of the state. The number of excess photons $\bar{n}_{\text {excess }}$ is just the total number of photons of both beams minus the number required to sustain the entanglement, and can be written in terms of the average of the amplitude and phase quadrature variances of the two entangled beams

$$
\begin{aligned}
\bar{n}_{\text {excess }} & =\bar{n}_{x}+\bar{n}_{y}-\bar{n}_{\min } \\
& =\left(\Delta^{2} \hat{X}_{x}^{+}+\Delta^{2} \hat{X}_{x}^{-}+\Delta^{2} \hat{X}_{y}^{+}+\Delta^{2} \hat{X}_{y}^{-}\right) / 4-\bar{n}_{\min }-1
\end{aligned}
$$

We characterized $\bar{n}_{\text {min }}$ and $\bar{n}_{\text {excess }}$ for our entanglement over the frequency range from $2.5 \mathrm{MHz}$ to $10 \mathrm{MHz}$. Measured spectra for $\Delta^{2} \hat{X}_{x \pm y}^{+}$and $\Delta^{2} \hat{X}_{x \pm y}^{-}$are shown in fig. 3 . The amplitude quadrature measurement is significantly degraded at low frequencies due to the resonant relaxation oscillation of our laser. Effectively this means that at low frequencies we have a mixed state with many more photons present than necessary for our entanglement. At high frequencies both spectra degrade due to the bandwidth limitations of our OPAs. Fig. 4 (a) shows the resulting spectra for $\sqrt{\Delta^{2} \hat{X}_{x \pm y}^{+} \Delta^{2} \hat{X}_{x \pm y}^{-}}$, this spectra directly results in a spectra for $\bar{n}_{\text {min. }}$. We measured the amplitude and phase quadrature variances of each entangled beam independently. The average of these measurements, which provides the total photon number $\bar{n}_{x}+\bar{n}_{y}$, is shown in fig. A(b).

$\bar{n}_{\text {min }}$ and $\bar{n}_{\text {excess }}$ were calculated from eqs. (6) and (7), and are plotted against each other in fig. 5. At low frequencies the number of excess photons is large due to contributions from our lasers resonant relaxation oscillation, and the number of photons required to maintain the entanglement is small due to poor squeezing. As the frequency increases away from the relaxation oscillation the number of excess photons decreases and the entanglement, and therefore $\bar{n}_{\text {min }}$, improves. At high frequencies the bandwidth of our OPAs limit the amount of squeezing and consequently the number of photons required to maintain the entanglement decreases. The contours on each of the four plots in fig. 5 show the efficacy of different protocols that the entanglement might be useful for. The details of how these contours were calculated will be included in a future publication [11]. Fig. 5(a) shows efficacy contours for demonstration of the EPR-paradox as defined in criterion (1), the curving of the contours shows the dependence of the criterion on mixedness. This plot implies that for our entanglement the optimum sideband frequency for observation of the EPRparadox is near 6.6 MHz. Fig. 5. (b) shows efficacy contours for unity gain quantum teleportation [10]. Here the contours run vertically, with no dependence on the mixedness of the entangled state, this result implies that optimum teleportation

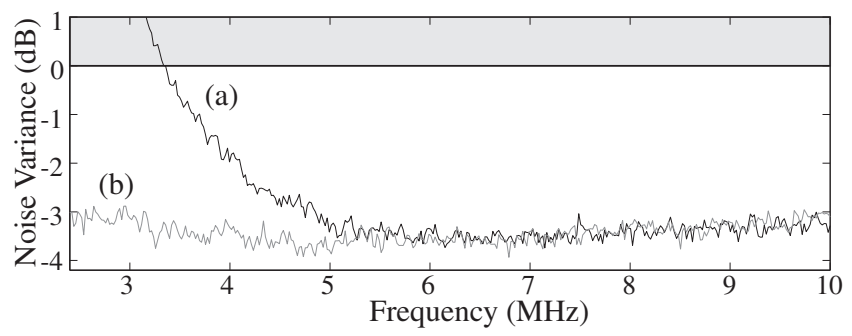

FIG. 3: Frequency spectra of (a) $\Delta^{2} \hat{X}_{x \pm y}^{+}$and (b) $\Delta^{2} \hat{X}_{x \pm y}^{-}$for our entangled beams with no additional loss. 


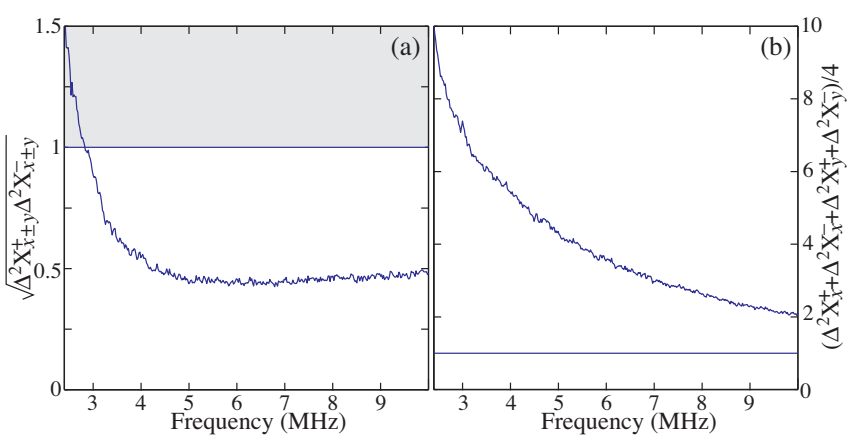

FIG. 4: Spectra of (a) $\sqrt{\Delta^{2} X_{x \pm y}^{+} \Delta^{2} X_{x \pm y}^{-}}$, and (b) the average of the quadrature noise variances of the individual entangled beams; with no additional loss.

results would be observed near $6.2 \mathrm{MHz}$. Fig. 5 (c) shows contours of the channel capacity for densecoding when an average of only 6.75 photons per bandwidth per time are allow in the sidebands. These contours show an extremely strong dependence on the number of excess photons carried by the entanglement, this is because every excess photon is one less that can be used to encode information. Accordingly, the optimum channel capacity would occur at $10 \mathrm{MHz}$ where our entanglement is most pure. Increasing the total number of photons allowed in the sidebands can negate this problem entirely [5]. In fig. 5. (d) we show efficacy contours for densecoding where 250 photons per bandwidth per time are allowed in the sidebands. In this case the contours are almost vertical and show very little dependence on the number of excess photons, resulting in an optimum channel capacity near $6.3 \mathrm{MHz}$.

In conclusion, we have produced strong, stably locked quadrature entanglement. It was characterized in terms of EPR and inseparability criteria with optimum results of $\Delta^{2} X_{x \mid y}^{+} \Delta^{2} X_{x \mid y}^{-}=0.58 \pm 0.02$ and $\sqrt{\Delta^{2} X_{x \pm y}^{+} \Delta^{2} X_{x \pm y}^{-}}=$ $0.44 \pm 0.01$, respectively. We observed qualitatively and quantitatively different behaviour for the two criteria on the introduction of controlled decoherence. A more complete characterization of entanglement can be achieved by considering both the inseparability and the mixedness of the entanglement. We introduce a photon number plot that characterizes the entanglement in terms of the number of photons necessary to maintain the entanglement, which can be directly related to the inseparability criterion, and the number of excess photons that contribute to mixedness. We represent our experimental results on such a diagram, and present efficacy contour for the EPR criterion, and teleportation and densecoding protocols.

[1] M. D. Reid and P. D. Drummond, Phys. Rev. Lett. 60, 2731 (1988); M. D. Reid, quant-ph/0112038.
[2] L-M. Duan et al., Phys. Rev. Lett. 84, 2722 (2000).

[3] C. H. Bennett et al., Phys. Rev. Lett. 70, 1895 (1993).

[4] C. H. Bennett and S. J. Wiesner, Phys. Rev. Lett. 69, 2881

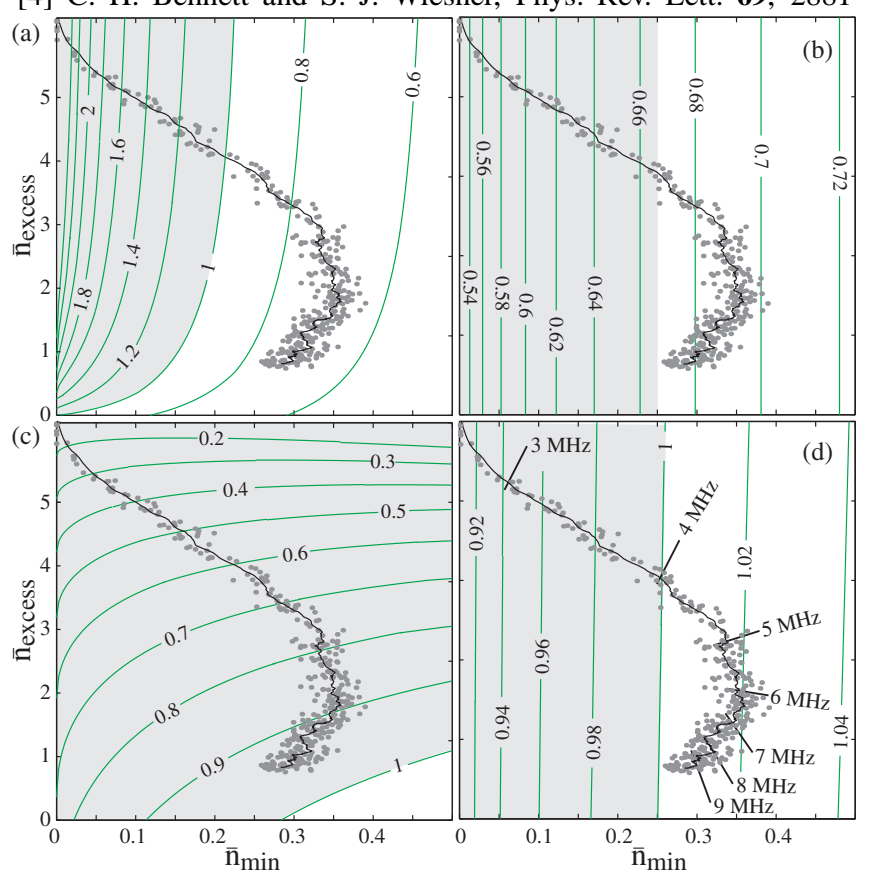

FIG. 5: Experimental results on photon number plots. The contours on the plots are (a) EPR criterion, (b) Fidelity measure of teleportation, (c) and (d) ratio of dense-coding channel capacity to optimum squeezed channel capacity for mean photon numbers of 6.75 and 250 , respectively.

(1992); S. L. Braunstein and H. J. Kimble, Phys. Rev. A 61, 042302 (2000).

[5] T. C. Ralph and E. H. Huntington, quant-ph/0208117.

[6] D. P. DiVincenzo, Science 270, 255 (1995).

[7] A. Einstein et al., Phys. Rev. 47, 777 (1935).

[8] W. P. Bowen et al., Phys. Rev. Lett. 88, 093601 (2002); R. Schnabel et al., quant-ph/0208103.

[9] See for example Z. Y. Ou et al., Phys. Rev. Lett. 68, 3663 (1992); Yun Zhang et al., Phys. Rev. A 62, 023813 (2000); and Ch. Silberhorn et al., Phys. Rev. Lett. 86, 4267 (2001).

[10] S. L. Braunstein and H. J. Kimble, Phys. Rev. Lett. 80, 869 (1998); and T. C. Ralph and P. K. Lam, Phys. Rev. Lett. 81, 5668 (1998).

[11] W. P. Bowen et al., Generation and characterization of quadrature entanglement yet to be written. 